\title{
Investigation of electron correlation effect on energy spectrum of two-electron systems in crystals with strong electron-phonon coupling
}

\author{
N.I.Kashirina, V.D.Lakhno*, V.V.Sychyov* \\ Institute of Semiconductor Physics, NAS of Ukraine, 45 prospect Nauky, 03028 Kyiv, Ukraine \\ E-mail: kashirin@class.semicond.kiev.ua \\ "Institute of Mathematical Problems of Biology, RAS, 142290 Pushchino, Russia \\ E-mail:lak@impb.psn.ru
}

\begin{abstract}
The influence of electron correlations (direct dependence of electronic wave function on distance between electrons) on energy spectra of two-electron systems $\left(D^{-}, F^{\prime}, F_{2}\right.$-centers and bipolarons) in polar crystals with strong electron-phonon interaction is investigated. Bipolaron is chosen as the simplest model of two-electron system in the crystal. Bipolaron energy is calculated for various distances between the centers of polarization wells of two polarons with accounting the electron correlations. A singlet bipolaron is stable at rather high energy of ion binding $\eta \leq \eta_{m} \approx 0,143\left(\eta=\varepsilon_{\infty} / \varepsilon_{0}\right)$. The unique energy minimum corresponds to a one-center bipolaron (an analog of a helium atom). The bipolaron binding energy constitutes up to $25.8 \%$ of a double polaron energy at $\eta \rightarrow 0$. A triplet bipolaron (an analog of orthohelium) is energetically disadvantageous. The one-center configuration of a triplet bipolaron corresponds to a maximum on the distance dependence of the total energy $J_{B p}(R)$. The exchange interaction between polarons has antiferromagnetic character. A prediction is made about a possibility of the Wigner crystallization of a polaron gas, which occurs with antiferromagnetic ordering in the polaron system. The examples of energy calculations with accounting the electron correlations of exchanged-coupled pairs, $D^{-}$and $F^{\prime}$-centers in polar crystals are also given.
\end{abstract}

Keywords: bipolaron, Frölich Hamiltonian, electron-phonon interaction, electron correlations, superconductivity, high-temperature superconductivity

Paper received 12.04.02; revised manuscript received 02.09.02; accepted for publication 10.12.02.

\section{Introduction}

Bipolaron is an elementary two-electron system in polar crystals. Such two-electron systems as for example $F^{\prime}$ and $F_{2}$-centers in polar crystals, and also $D^{-}$-centers and exchange-coupled pairs of paramagnetic centers $(\mathrm{PC})$ in covalent crystals and crystals with mixed polar -covalent coupling can be considered as bound bipolaron placed in a field of static charges. For this reason bipolaron is the simplest test system to check the methods of an energy electron systems accounts in a solids, to which interaction of an electron subsystem with a field of crystal elementary excitations are essential. The optical phonons field (interaction with which is described by Frölich Hamiltonian) is one of examples of such numerous fields (acoustic phonons, plasmons, spin waves, waves of charge density, etc.).

The bipolaron problem has revived again due to the discovery of a high-temperature superconductivity (HTSC). Unfortunate numerical errors made in early papers devoted to bipolarons (see, for example, review
[1]) were corrected in [2]. The energy minimum obtained in [2] for a one-center bipolaron (or Pecar bipolaron[3]) was much lower than a corresponding minimum originally found in [4] for a two-center bipolaron. In terms of spatial configuration, the one-center bipolaron is analogous to a helium atom, while the two-center one is an analog of a hydrogen molecule. Subsequently, the results of [2] were repeatedly reproduced for both Slater and Gaussian functions [1, 5-7].

The deciding factor, when choosing a bipolaron probe wave function (WF) in variational calculations, was its nonmultiplicative form. For the two-center bipolaron the probe WF was chosen [4] as a symmetrized product of one-electron WFs. For a one-center bipolaron correlation effects were taken into account by introducing a term depending on the difference in electron coordinates $[2,5$, 6] (in what follows, this particular type of correlation effects will be referred to as electron correlations).

However, until the present time no calculations have been performed to take into account both these effects at the same time. Therefore, the question which of the two 
bipolaron configurations is energetically more advantageous still remains open. Thus, the works [8,9] continue to explore the two-center bipolaron (despite the fact that the author of [2] obtained a deeper minimum for the onecenter bipolaron than in $[8,9])$. A quantitative estimate of the contribution of electron correlations into the energy of two-electron systems of the type of large-radius paired centers in polar crystals $\left(\mathrm{F}_{2}\right.$-center or "a hydrogen molecule placed in a phonon field") is yet to be obtained, too. To solve the problems of this sort, one should simultaneously consider both the permutation symmetry of WF and the electron correlation.

The energy of the one-center bipolaron can be calculated using wave functions of the Pecar type [3]

$$
\begin{aligned}
& \Psi\left(\mathbf{r}_{1}, \mathbf{r}_{2}\right)= \\
& =N\left(1+\gamma r_{12}\right)\left(1+\alpha r_{1}\right)\left(1+\alpha r_{2}\right) \exp \left(-\alpha\left(r_{1}+r_{2}\right)\right),
\end{aligned}
$$

where $N$ is a normalization multiplier, $r_{1}, r_{2}$ are the coordinates of the first and second electrons, respectively, $r_{12}=\left|r_{1}-r_{2}\right|$ is the distance between the first and the second electron, $r_{1}=\left|r_{1}\right|, r_{2}=\left|r_{2}\right|, \gamma$ and $\alpha$ are variational parameters.

The bipolaron energy is calculated analytically and the relevant functional is subsequently varied over the parameter $\gamma(\alpha$ is uniquely determined by $\gamma)$. The deciding factor in the formation of a bound two-electron state is the availability of a correlation term proportional to the difference in electron coordinates $\gamma r_{12}$.

Calculations of the two-center bipolaron are concerned with consideration of the permutation symmetry of the electron system. The wave function of the two-electron system is chosen in the symmetrized form:

$$
\Psi\left(\mathbf{r}_{1}, \mathbf{r}_{2}\right)=\Phi\left(\mathbf{r}_{1}, \mathbf{r}_{2}\right)+\Phi\left(\mathbf{r}_{2}, \mathbf{r}_{1}\right) .
$$

In early papers devoted to calculation of bipolaron states, $\Phi\left(\mathbf{r}_{1}, \mathbf{r}_{2}\right)$ was chosen as a product of hydrogen-like WF centered at various points [4]. In the recent paper [8] that deals with calculation of a two-center bipolaron, $\Phi\left(\mathbf{r}_{1}\right.$, $\mathbf{r}_{2}$ ) is chosen as a product of the Pecar polaron functions $\left(1+\alpha r_{a 1}\right) \exp \left(-\alpha r_{a 1}\right)\left(1+\alpha r_{b 2}\right) \exp \left(-\alpha r_{b 2}\right)$ (where use is made of the notation traditional for the two-center coordinate system: $r_{a 1}\left(r_{a 2}\right)$ is the radius-vector of the first (second) electron reckoned from the center $a, r_{b 1}\left(r_{b 2}\right)$ is the same for the center $b$ ). Both in [4] and in [8] the energy minima were much higher than a corresponding minimum obtained in [2] with the use of the wave function (1). The essence of variational calculations suggests that preference should be given to the WF that gives a deeper energy minimum. However, final conclusions of which of two bipolarons is more preferable energetically can be made only in the case when the electron correlations in the wave function (2) are taken into account. This means the choice

$$
\begin{aligned}
& \Phi\left(\mathbf{r}_{1}, \mathbf{r}_{2}\right)= \\
& =\left(1+\alpha r_{a 1}\right)\left(1+\alpha r_{b 2}\right)\left(1+\gamma r_{12}\right) \exp \left(-\alpha\left(r_{a 1}+r_{b 2}\right)\right) .
\end{aligned}
$$

However, though the wave function (3) has rather simple form, one runs into severe obstacles when trying to find analytically the two-center integrals. Therefore, the problem of a two-center bipolaron cannot be solved in the way as it was done for the one-center bipolaron. But if WF are chosen in the form of a combination of Gaussian orbitals, analytical calculations become straightforward and the problem is reduced to variation of a multiparametrical functional.

Recently interest in polaron and bipolaron subject field has surfaced again due to expansion of this field of investigations to anisotropic crystals, low-dimensional structures and systems with quantum wells [6, 10-13]. The problem of the choice of a bipolaron spatial configuration and consideration of electron correlations is actual for such systems, too.

The aim of this work was to calculate the bipolaron energy depending on the distance between the centers of polarization wells taking into account both electron correlations and the permutation symmetry of the electron system.

In spite of the fact that bipolaron is the elementary two-electron system in polar crystals, its functional includes the most complex for the explanation part corresponding to nonlocal potential describing the interaction of the electron system with a phonon field. The given interaction has no analogs in the nuclear and molecular systems. From this point of view the description of the bipolarons bound with a field of static charges only insignificantly complicates a task. Therefore, we examine a general case of this bound bipolaron and execute specific numerical accounts both for free and the bound states.

\section{Main formulae and relations}

Let us choose the Hamiltonian of a system consisting of two electrons and a phonon field in the form:

$$
\begin{aligned}
& \mathbf{H}=\hbar \omega \sum_{k} a_{k}^{+} a_{k}+ \\
& +\sum_{k} V_{k}\left(a_{k}-a_{-k}^{+}\right)\left(\exp \left(i \mathbf{k} \mathbf{r}_{1}\right)+\exp \left(i \mathbf{k r} \mathbf{r}_{2}\right)\right)- \\
& -\frac{\hbar^{2}}{2 m^{*}} \Delta_{1}-\frac{\hbar^{2}}{2 m^{*}} \Delta_{2}+\frac{e^{2}}{\varepsilon_{\infty}\left|\mathbf{r}_{1}-\mathbf{r}_{2}\right|}+\mathbf{H}_{C}
\end{aligned}
$$

where $V_{k}=-i \frac{e}{k} \sqrt{\frac{2 \pi \hbar \omega}{V \tilde{\varepsilon}}}, \frac{1}{\tilde{\varepsilon}}=\frac{1}{\varepsilon_{\infty}}-\frac{1}{\varepsilon_{0}}, V$ is the crystal volume, $\omega$ is the frequency of optical phonons, $\mathbf{k}$ is the wave vector of the phonons, $a_{k}^{+}$and $a_{k}$ are the operators of creation and annihilation of phonons with the wave vector $\mathbf{k}, \varepsilon_{\infty}$ and $\varepsilon_{0}$ are the high-frequency and static dielectric constants respectively, $r_{1}, r_{2}$ are electron coordinates. The first term in (4) corresponds to Hamiltonian of the field of optical phonons, the second one represents Hamiltonian of electron-phonon interaction in a two-elec- 


\section{N.I.Kashirina et al.: Investigation of electron correlations effect on energy spectrum...}

tron system written in the Frölich form, the third and the fourth terms stand for kinetic energy of electrons, the fifth term describes Coulomb repulsion of electrons, $\mathrm{H}_{\mathrm{c}}$ describes all Coulomb interactions of electrons with static charges and those among themselves.

Canonical transformation of Hamiltonian (4) $\exp \left(S_{a}\right) H \exp \left(-S_{a}\right)$, with $S_{a}=\sum_{k} C_{k}\left(a_{k}^{+}-a_{k}\right)$ and variation over parameters $C_{k}$ (shift transformation) yields the following expression for the functional of the bipolaron ground state

$$
\begin{aligned}
& S_{a}=\sum_{k} C_{k}\left(a_{k}^{+}-a_{k}\right) \\
& \bar{V}_{e e}=\left\langle\Psi\left(\mathrm{r}_{1}, \mathrm{r}_{2}\right)\left|\frac{e^{2}}{\varepsilon_{\infty} r_{12}}\right| \Psi\left(\mathrm{r}_{1}, \mathrm{r}_{2}\right)\right\rangle, \\
& \bar{H}_{C}=\left\langle\Psi\left(\mathrm{r}_{1}, \mathrm{r}_{2}\right)\left|\mathrm{H}_{C}\right| \Psi\left(\mathrm{r}_{1}, \mathrm{r}_{2}\right)\right\rangle \\
& \bar{T}=-\frac{\hbar^{2}}{2 m^{*}}\left\langle\Psi\left(\mathrm{r}_{1}, \mathrm{r}_{2}\right)\left|\Delta_{1}+\Delta_{2}\right| \Psi\left(\mathrm{r}_{1}, \mathrm{r}_{2}\right)\right\rangle \\
& U_{k}=V_{k}\left\langle\Psi\left(\mathrm{r}_{1}, \mathrm{r}_{2}\right)\left|\exp \left(i \mathrm{kr}_{1}\right)+\exp \left(i \mathrm{kr}_{2}\right)\right| \Psi\left(\mathrm{r}_{1}, \mathrm{r}_{2}\right)\right\rangle
\end{aligned}
$$

In what follows we will use effective atomic units putting $\hbar=1, e=1, m^{*}=1, \varepsilon_{\infty}=1$, i.e. the unit of energy will be $R y^{*}=e^{4} m^{*} / \hbar^{2} \varepsilon_{\infty}^{2}$ and the unit of length will be effective Bohr's radius $a_{0}^{*}=\hbar^{2} \varepsilon_{\infty} / m^{*} e^{2}$.

Summing functional (5) over the wave vector and integrating it we express it in the form traditional for bipolaron problems:

$$
\begin{aligned}
& J_{B p}=\bar{T}+\bar{V}_{e e}+\bar{H}_{C}+\bar{V}_{e f}, \\
& \bar{V}_{e f}=-\frac{2 e^{2}}{\tilde{\varepsilon}} \int \frac{\left|\Psi\left(\mathrm{r}_{1}, \mathrm{r}_{2}\right)\right|^{2}\left|\Psi\left(\mathrm{r}_{3}, \mathrm{r}_{4}\right)\right|^{2}}{r_{13}} d \tau_{12} d \tau_{34} .
\end{aligned}
$$

Note, that in this formulation of the problem we do not use the concept of adiabatic approximation anywhere. We perform variational calculations of the initial Hamiltonian (4) which after canonical transformation changes to functional (5). Transformation of this sort leads to the results of strong-coupling limit and gives the first term in the expansion of the polaron (bipolaron) energy in terms of $1 / \alpha$ (where $\alpha$ is the Frölich binding constant, $\alpha>>1$ ) proportional to $\alpha^{2}$,

$\alpha=\frac{e^{2}}{\hbar \tilde{\varepsilon}} \sqrt{\frac{m^{*}}{2 \hbar \omega}}$.

The probe WF will be chosen as a linear combination of Gaussian orbitals:

$$
\Psi_{S}\left(\mathrm{r}_{1}, \mathrm{r}_{2}\right)=\Phi\left(\mathrm{r}_{1}, \mathrm{r}_{2}\right)+(-1)^{S} \Phi\left(\mathrm{r}_{2}, \mathrm{r}_{1}\right),
$$

where $S=0$ for the singlet state (symmetric with respect to the operation of permutation of electron coordinates) and $S=1$ for the triplet (antisymmetric) bipolaron state.
In turn

$\Phi\left(\mathrm{r}_{1}, \mathrm{r}_{2}\right)=$

$=\sum_{i=1}^{n} C_{i} \exp \left(-a_{1 i} r_{a 1}^{2}-2 a_{2 i}\left(\mathrm{r}_{1} \cdot \mathrm{r}_{2}\right)-a_{3 i} r_{b 2}^{2}\right)$

where $r_{1}, r_{2}$ is the radius-vector of the first (second) electron reckoned from the coordinate origin placed halfway between the points $a$ and $b$. The axis $O z$ is directed from the center $a$ to the center $b$. The distance between the points $a$ and $b$ is equal to $R$. The quantities $C_{\mathrm{i}}, a_{1 \mathrm{i}}, a_{2 \mathrm{i}}, a_{3 \mathrm{i}}$ are variational parameters. The electron correlations are taken into account by the term $2 a_{2 \mathrm{i}}\left(\mathbf{r}_{1} \cdot \mathbf{r}_{2}\right)$ in (14), $\left(\mathbf{r}_{1} \cdot \mathbf{r}_{2}\right)$ is the scalar product of vectors $\mathbf{r}_{1}$ and $\mathbf{r}_{2}$.

Polaron WF is chosen in the form:

$\Psi_{p}(\mathrm{r})=\sum_{i=1}^{n} c_{i} \exp \left(-\alpha_{i} r^{2}\right)$,

where $C_{\mathrm{i}}, \alpha_{\mathrm{i}}$ are variational parameters.

On proceeding to the one-center bipolaron at $a_{1 \mathrm{i}}=a_{3 \mathrm{i}}$ WF (14) is equivalent (with an accuracy of the notation of variational parameters) to the WF used in [5] for the one-center bipolaron and in the limit case of the absence of anisotropy coincides with the wave function suggested in [6] for calculation of the energy of the one-center bipolaron in anisotropic crystals.

\section{Calculation results}

\subsection{Singlet bipolaron}

Qualitative analysis of correlation effects can conveniently be carried out with the use of a simplest three-parameter wave function:

$\Phi\left(\mathrm{r}_{1}, \mathrm{r}_{2}\right)=\exp \left(-a_{1} r_{a 1}^{2}-2 a_{2}\left(\mathrm{r}_{1} \cdot \mathrm{r}_{2}\right)-a_{3} r_{b 2}^{2}\right)$.

Fig.1 shows the dependencies of the energy of the ground (singlet) state on the distance between the centers of polarization wells of two polarons. It is seen that as the distance between the polarons increases the functional of the bipolaron system breaks down into functionals corresponding to the two polarons, the bipolaron energy tends to doubled polaron energy calculated in this approximation $(1 / 3 \pi)$, and the importance of electron correlations decreases. The Fig. 1 also displays the dependencies of corresponding energies for the system without regard for electron correlations (curves 1 and 2). In going to the multiplicative form at $R=0$ (curve 1 is calculated for $a_{1}=a_{3}$, $a_{2}=0$ and corresponds to symmetrized product of Gaussian orbitals), the bipolaron functional breaks down into doubled polaron functional. The choice of WF in the more general form $\left(a_{1} \neq a_{3}, a_{2}=0\right)$ leads to arising of two minima, one of which (less deep) corresponds to the one-center bipolaron and the other - to the two-center one (curve 2). Taking into account electron correlations results in a unique minimum at $R=0$, which corresponds to the one-center bipolaron (curve 3 calculated for $\left(a_{1}=\right.$ 
N.I.Kashirina et al.: Investigation of electron correlations effect on energy spectrum...

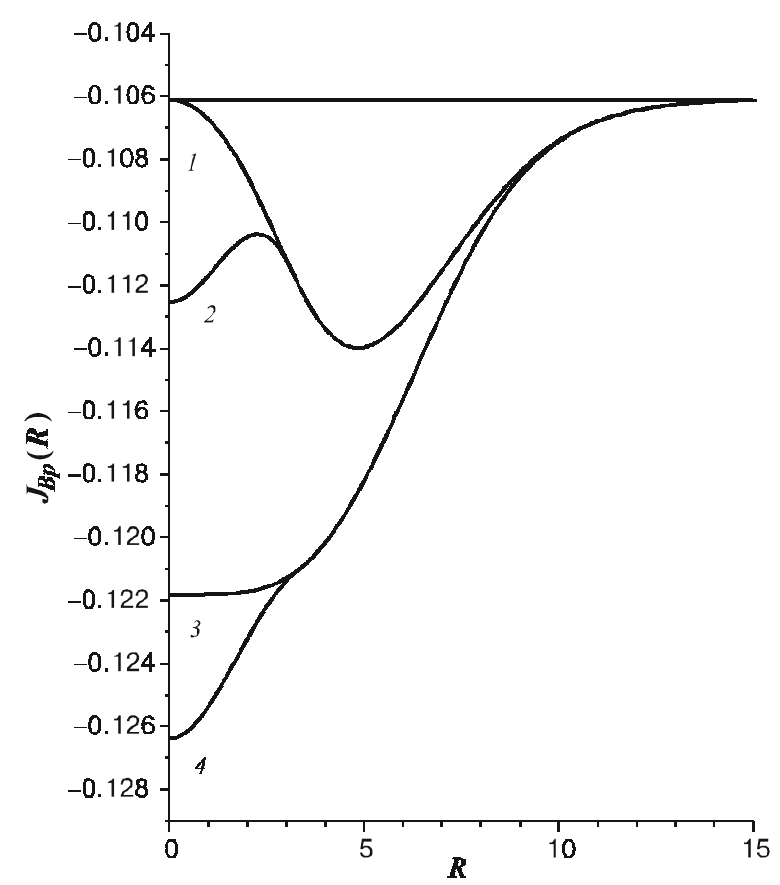

Fig. 1. Dependence of the bipolaron energy on the distance between centers of polarization wells for one exponent $(n=1)$ : curve 1 corresponds to one variational parameter $\left(a_{1 \mathrm{i}}=a_{3 \mathrm{i}}\right.$, $a_{2 \mathrm{i}}=0-$ electron correlations are absent), curve 2 is constructed for two variational parameters $\left(a_{1 \mathrm{i}}, a_{3 \mathrm{i}}, a_{2 \mathrm{i}}=0\right.$ - electron correlations are absent), curve 3 - two variational parameters $\left(a_{1 \mathrm{i}}=a_{3 \mathrm{i}}, a_{2 \mathrm{i}}\right)$, curve $4-$ three variational parameters $\left(a_{1 \mathrm{i}}, a_{3 \mathrm{i}}, a_{2 \mathrm{i}}\right)$

$\left.=a_{3}, a_{2} \neq 0\right)$ ). For the three-parameter WF (curve 4 calculated for $a_{1} \neq a_{3}, a_{2} \neq 0$ ), the deepest and unique minimum corresponds to $R=0$ (the one-center bipolaron), so the two-center bipolaron obtained without regard for electron correlations is associated with less appropriate choice of WF.

As the number of exponents in expression (14) increases, the qualitative picture shown in Fig. 1 does not change. Fig. 2 presents the dependencies of the bipolaron energy for five exponents on the distance between the centers of polarization wells of two polarons (with (2) and without (1) regard for electron correlations). As comparison of Figs 1,2 suggests, the qualitative picture of the relation between the values of the energy minima for the one-center and two-center bipolarons does not change, though the bipolaron coupling energy grows more than twice. Further increase in the number of exponents in the initial wave function does not lead to any noticeable qualitative changes. The unique energy minimum corresponds to the one-center configuration as earlier.

Fig. 3a shows the dependencies of various contributions into the bipolaron energy (kinetic energy, energy of electron-electron interaction, phonon contibution into the total energy) for five terms of the wave function (14). In Fig. $4 \mathrm{~b}$ the appropriate dependencies without the account of electron correlations for wave functions, chosen as a symmetrized product of polaron $\operatorname{VF}\left(a_{1 \mathrm{i}}=a_{3 \mathrm{i}}\right.$, $a_{2 \mathrm{i}}=0$ ) are shown.

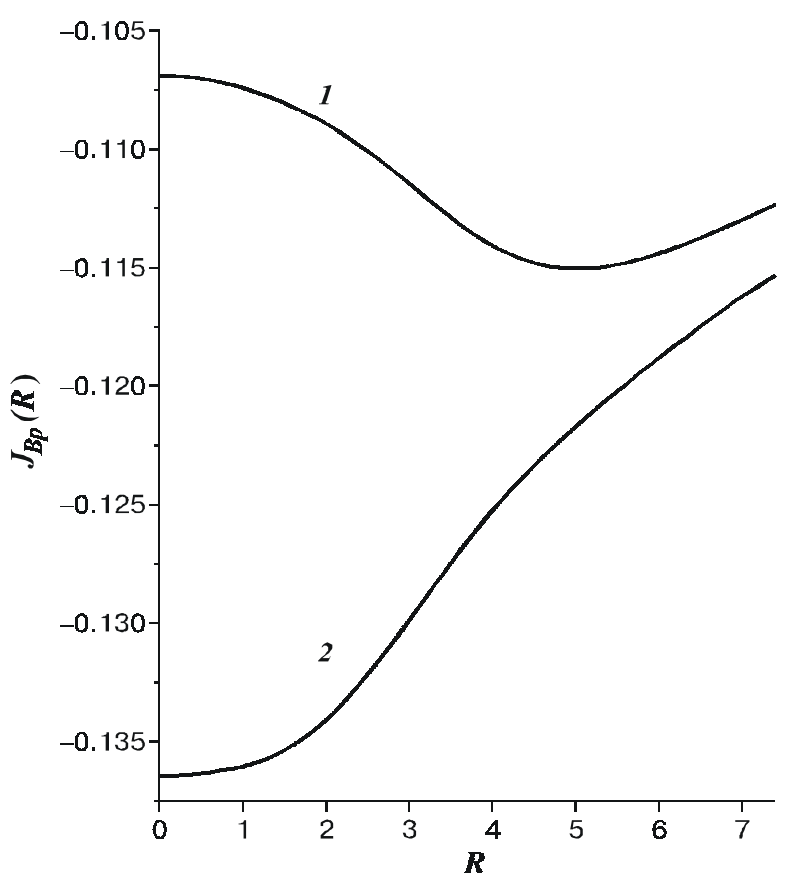

Fig. 2. Distance dependence of the bipolaron energy: curve 1 without regard for the correlation $\left(a_{1 \mathrm{i}}=a_{3 \mathrm{i}}, a_{2 \mathrm{i}}=0, n=5\right)$, curve 2 - the most general function (variational parameters $a_{1 \mathrm{i}}, a_{3 \mathrm{i}}, a_{2 \mathrm{i}}$, $n=5)$.

So, the two-center bipolaron is energetically disadvantageous in an isotropic crystal, and the energy minimum arising at $R>0$ is associated with inappropriate choice of WF without due regard for electron correlations.

In many papers, relative values of the bipolaron binding energy are given (in the units of polaron energy calculated in the same approximation), which leads to misunderstanding in comparing the values of energy minima obtained by different authors. To remedy this, we give absolute values of the energy found using various WF.

The Pecar function (1) at $\eta=\varepsilon_{\infty} / \varepsilon_{0}=0$ leads to the ground state energy $J_{B p}=-0.1346292$.

The polaron binding energy is given by

$\Delta E=J_{B p}-2 J_{p}$

Or, in relative units $\Delta E / 2 J_{p} \approx 0.241$, where $J_{p}=$ $=-0.0542564$ is the exact value of the polaron energy obtained numerically in the limit of strong binding in [14]. This value of $J_{p}$ with all the significant digits is found using the polaron WF in the form (15) for $n=5$. The region of existence of the bipolaron for the Pecar WF is determined by $\eta=\varepsilon_{\infty} / \varepsilon_{0} \leq \eta_{m}=0.132$.

In [2] absolute values of the ground state energy are not given, and slightly overestimated results for the binding energy and the region of existence of the bipolaron $\left(\Delta E / 2 J_{p} \approx 0.25, \eta_{m} \approx 0.14\right)$ were associated with the fact that the bipolaron energy was calculated with respect to slightly overestimated value of polaron energy obtained for WF chosen in the form $(1+\alpha r) \exp (-\alpha r)$. 
N.I.Kashirina et al.: Investigation of electron correlations effect on energy spectrum...
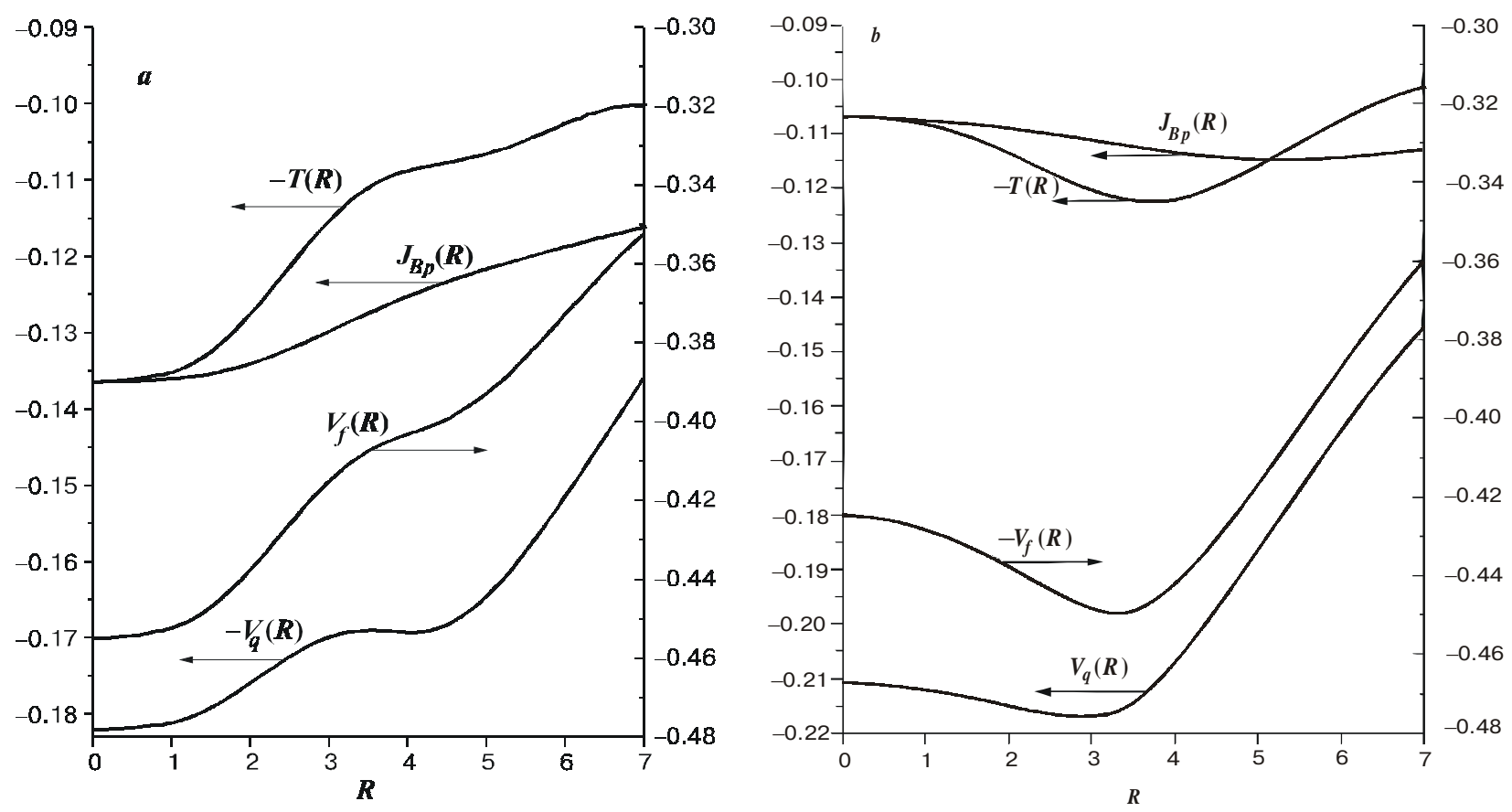

Fig.3. $a$ - Dependencies of various contributions into the bipolaron energy on the distance between the centers of polarization wells. $T(R)$ is the bipolaron kinetic energy, $V_{q}(R)$ is the energy of interelectron interaction, $V_{f}(R)$ is the phonon contribution into the bipolaron energy $\left(T(R)>0, V_{q}(R)>0, V_{f}(R)>0\right) . J_{B p}(R)$ is the total bipolaron energy. The calculations are performed for variational parameters $a_{1 \mathrm{i}}, a_{3 \mathrm{i}}, a_{2 \mathrm{i}}, n=5 ; \quad b$ - the appropriate dependencies without the account of electron correlations for wave functions, chosen as symmetrized product of polaron $\operatorname{VF}\left(a_{1 \mathrm{i}}=a_{3 \mathrm{i}}, a_{2 \mathrm{i}}=0\right)$.

The best value of the energy of the bipolaron ground state obtained by us at $\eta=0(n=11)$ was $J_{p}=-0.136509$, or in dimensionless units $\Delta E / 2 J_{p} \approx 0.258, \eta_{m} \approx 0.143$.

In the strong-coupling regime, the present approach provides a larger binding energy and broader region of the bipolaron stability then those obtained in $[2,4,5,8,15]$.

Fig. 4 shows the dependencies $\Delta \mathrm{E} / \mathrm{R}$ (singlet state) for different $\eta$.

\subsection{Triplet bipolaron}

In studies of photoconductivity $\sigma(\omega)$ in YBCO Deving and Salje [16] found a wide absorption peak in the infrared frequency range $\left(\omega \approx 5.5 \cdot 10^{3} \mathrm{~cm}^{-1}\right)$. According to their interpretation, the peak corresponds to the transition of bosons from the ground (singlet) to the excited metastable triplet state. These authors proposed that at rather low temperatures singlet bipolarons coexist with triplet ones, and just triplet bipolarons are responsible for widening of $\mathrm{Cu}$ and $\mathrm{O}$ NMR lines in YBCO. In this case, the population of triplet levels should increase with temperature so that at $T \approx 200 \mathrm{~K}$ just triplet bipolarons should have a dominant role in conductivity. Subsequently this predicted in [16] change in the shape of the curve $\rho$ $T$ was actually observed at that temperature (see review [17] and references therein).

We have calculated the energy of a triplet bipolaron (analog of ortho-helium). The best numerical value obtained for the energy of a one-center configuration of triplet bipolaron using of $\mathrm{WF}(13)$ at $S=1, \eta=0$ was $J_{p}=-0.076072$.

As the distance between the centers of polarization wells increases, the energy corresponding to the triplet

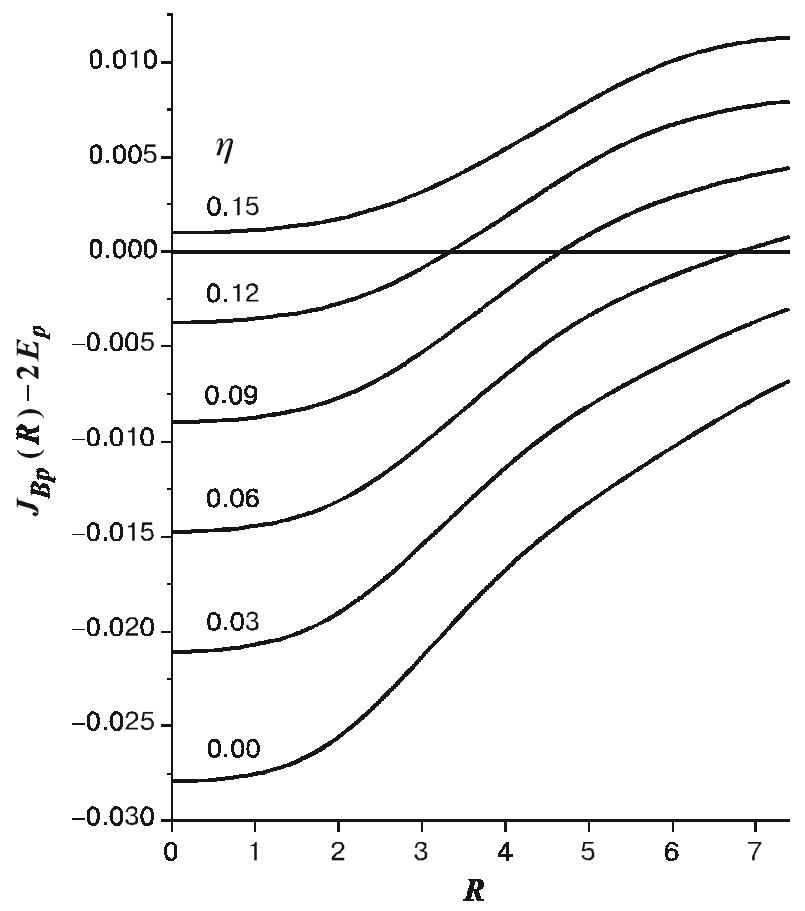

Fig. 4. The bipolaron binding energy as a function of distance between the centers of polarization wells for various parameters $\eta$. 
term of bipolaron monotonically decreases (from -0.76072 at $R=0$ ) by complete analogy with ${ }^{3} \mathrm{~S}_{\mathrm{u}}$ - term of a hydrogen molecule. At $R=0$ the distance dependence of the energy exhibits rather a sharp maximum, which suggests instability of a triplet $2^{3} \mathrm{~S}$ state that can arise, for example, under nonequilibrium conditions during exchage scattering at bipolarons of zone electrons. In this case, bipolarons break down into isolated polarons.

Note also that the use of WFs (13), (14) for the calculation of the energy of pair- and orthohelium has enabled us to get full agreement with experimental results, which suggests considerable flexibility of the WFs used.

\subsection{Exchange interaction between polarons}

Noteworthy is the occurrence of antiferromagnetic (AF) exchange interaction between polarons. At rather large distances, WF of a polaron can be presented as a symmetrized (singlet state) or antisymmetrized (triplet state) product of the polaron WF. Then the interaction energy of two polarons has the form (with an accuracy of the terms quadratic in overlapping integral $\mathrm{K}$ )

$$
E_{\text {int }}=E_{1}-J_{e x} \mathrm{~S}_{1} \mathrm{~S}_{2}
$$

where $\mathbf{S}_{1}$ and $\mathbf{S}_{2}$ are spins of the first and the second electron,

$$
\begin{aligned}
& E_{1}=\frac{1}{\varepsilon_{0}} \int \frac{a(1)^{2} b(2)^{2}}{r_{12}} d \tau_{12}, \\
& J_{e x}=\frac{1}{\varepsilon_{\infty}} K_{1}-\frac{4}{\tilde{\varepsilon}} K_{2} K, \\
& K_{1}=\int \frac{a(1) b(1) a(2) b(2)}{r_{12}} d \tau_{12}, \\
& K_{2}=\int \frac{a(1) b(1) b(2)^{2}}{r_{12}} d \tau_{12}, \\
& K=\int a(1) b(1) d \tau_{1},
\end{aligned}
$$

where we use the notation traditional for two-center coordinate systems: $a(1), b(1)$ are the WFs of a polaron centered at points $a$ and $b$, respectively $\left(a(1) \equiv \Psi_{p}\left(r_{a 1}\right)\right.$, $\left.b(1) \equiv \Psi_{p}\left(r_{b 1}\right)\right)$.

The first term in (20) corresponds to the ferromagnetic Coulomb exchange, the second one describes antiferromagnetic (AF) interaction between polarons caused by phonon contribution.

Thus, at large distances (if $\varepsilon_{0}>>\varepsilon_{\infty}, J_{e x} \approx-3 K^{2} / \varepsilon_{\infty} R$ ), polarons push off and the spin-depending part of the interaction (total exchange) has AF character. Therefore, the Wigner crystallization accompanied by AF ordering can occur in a polaron system.

It also follows that for a bipolaron state to be formed the potential barrier should be overcome.
AF interaction between polarons can be responsible for the decrease in the paramagnetic component in the magnetic susceptibility of a polaron gas as the polaron concentration increases even though bound bipolaron states are not formed.

Instability of triplet bipolaron with respect to its breaking down into individual polarons, obtained by us in the framework of Frцlich Hamiltonian describing the interaction of electrons with optical phonons, does not exclude a possibility for the formation of triplet states in a two-electron system caused by the interaction of electrons with elementary excitations of different nature, for example, with spin waves. Formation of self-localized electron states in antiferromagnetics with the low Neel temperature ("spin polaron" in an AF crystal) was considered in $[18,19]$. The authors of [17] proposed that high temperature superconductivity is associated just with spin polarons that form a bound state by full analogy with a bipolaron.

\section{Bound bipolarons}

As an example of bound bipolarons we shall consider the exchange coupled pairs of paramagnetic centers (PC) in polar crystals. For such system the part of Hamiltonian, describing interaction of an electron subsystem with a field of Coulomb charges, has the following form:

$$
\mathrm{H}_{k}=-\frac{e^{2}}{\varepsilon_{0} r_{a 1}}-\frac{e^{2}}{\varepsilon_{0} r_{a 2}}-\frac{e^{2}}{\varepsilon_{0} r_{b 1}}-\frac{e^{2}}{\varepsilon_{0} r_{b 2}}+\frac{e^{2}}{\varepsilon_{0} R},
$$

where the first and the second terms describe interaction of the first and the second electron with a charge localized in a point (a), and the third and fourth ones describe interaction the first and the second electron with a charge localized in a point (b), correspondingly, the fifth term describes interaction of static charges among themselves.

The configuration of considered system corresponds to that of the molecule of hydrogen "placed in phonon field". In contrast with the molecular systems, the distance between PC in a crystal are determined by a structure of a crystal lattice, instead of distance $R_{m}$, corresponding to a minimum of the functional of the exchange coupled pairs. For the same reason, in contrast with the molecular systems, the triplet terms (for which on the curve dependence of the energy from a distance between PC a minimum is not present, as, for example for the triplet ${ }^{3} \mathrm{~S}_{\mathrm{u}}$-term) are observable.

To estimate the role of the correlation effects for the bound two-electron states, we shall choose as an example the exchange coupled pairs of PC in a crystal. In Fig. 5 the dependences of energy of the ground state of the exchange coupled pairs of PC for parameters of metal-ammonia solution are presented. The curve (1) corresponds to the consideration performed without any account of electron correlations (hydrogen-like WF), the curve (2) corresponds to the energy of exchange coupled pairs designed in view of electronic correlations for $n=5$ in ex- 


\section{N.I.Kashirina et al.: Investigation of electron correlations effect on energy spectrum...}

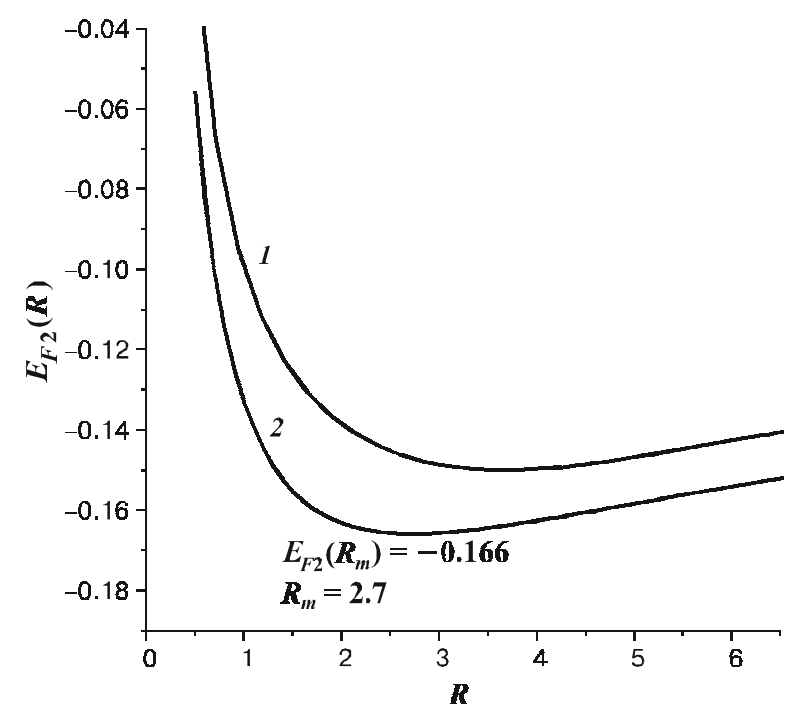

Fig. 5. Dependencies of energy of the ground state of the exchange coupled pairs of PC for parameters of metal-ammonia solution, curve (1) corresponds to the consideration performed without account of electron correlations (hydrogen-like WF), the curve (2) corresponds to the energy of exchange coupled pairs designed in view of electronic correlations for $n=5$ in expression (14).

pression (14). The comparison of the size of the power minimum shows that the account of correlation effects in crystals with ion coupling lowers it down to $12 \%$ in comparison with the appropriate value calculated without consideration of electron correlations. Notice for comparison that in the atomic and molecular systems the given value does not exceed $0.1 \%$. Thus, the most sensitive to the account of electron correlations is the unlocal part of two-electron system functional in a crystal describing electron interaction with a phonon field. Let's present also following values: the energy $F_{2}$-center in metal- ammonia solution is -0.166 , the energy of $F^{\prime}$-center 0.1535 , the bipolaron energy -0.1028 , the polaron energy -0.0459 , the energy of $F$-center -0.0723 correspondingly. The value -0.093 corresponds to the triplet state of $F^{\prime}$-center, i.e. the given state in considered system is energetically unstable, as well as the triplet bipolaron.

\section{Conclusions}

In the work, the study of influence of electron correlations on a power spectrum of two-electronic systems in a crystal is carried out. As the model system the crystals with strong electron-phonon interaction are chosen. The research of influence of electron correlations on a spatial configuration of the bipolaron in low-dimensional systems deserves independent consideration.

The results obtained will be generalized to crystals with intermediate electron-phonon coupling in a special paper. Notice, only, that the conclusion about the energetic disadvantage of a two-center bipolaron remains unchanged. Formal generalization of the results obtained by the strong coupling method to the range of intermediate values of the Frulich electron-phonon interaction parameter $\alpha$ using the formula

$$
J_{i}=2 J_{B p} \frac{\alpha^{2}}{(1-\eta)^{2}} \hbar \omega,
$$

(where $J_{B p}$ corresponds to the bipolaron energy in the strong coupling limit in effective Rydberg $R y^{*}=e^{4} m^{*} / \hbar^{2} \varepsilon_{\infty}^{2}$ ), naturally gives underestimated values for the bipolaron energy and the critical parameter $h_{c}$ as compared to the results obtained by the intermediate coupling method [15, $21]$. Thus, the lowest values of the bipolaron energy for $\alpha<8$ are obtained in [20] where at $\alpha=7, \eta=0, J_{B p}$ is calculated to be $-16.28 \hbar \omega$, while formula (24) yields $13.38 \hbar \omega$.

Notice that the variation parameter $a=\left\langle r_{1}-r_{2}\right\rangle$ which is treated in [20] as the mean distance at which electrons fluctuate is analogous to the variation parameter R used in this paper. Our results correlate well with the fact that the only energy minimum of a bipolaron corresponds to $a=0$ [20].

In the range $8<\alpha<25$, the lowest value of the bipolaron functional corresponds to the results of [15] and for $25<\alpha$ the upper bound of the bipolaron energy is determined by the formula (24).

In spite of the fact that the study of influence of electronic correlations on a spatial of bipolaron configuration, carried out by us, has shown that two - center bipolaron is energetically unprofitable, we shall notice that the first works devoted to study of the bipolaron and which have proved a basic opportunity of its formation were executed just for the two-center one [4]. And in [21], also executed for the two-center configuration, it was shown for the first time that the transition to anisotropic systems results in stabilization of bipolaron. This circumstance, naturally, allocates works $[4,21]$ in a number of the subsequent works devoted to study of various aspects of the theory of bipolarons as basic ones, in spite of the fact that trial VF were chosen not by the most successful image.

At the same time the account of an oscillatory spectrum of the bipolaron in the vicinity of nonexistent minimum, appropriates the two-center configuration of bipolaron [9] is an example of study of a fictitious effect, existence of which is impossible even to suppose.

\section{Acknowledgments}

The work was supported by Russian Foundation for Basic Research. Project code 01-07-90317.

\section{References}

1. V.L. Vineckiy, N.I. Kashirina, E.A. Pashickiy, Bipolaron states of great radius and the problem of high temperature superconductivity // Ukrainsk. Fiz. Zhurn. 37(1), pp.76- 94 (1992). 


\section{N.I.Kashirina et al.: Investigation of electron correlations effect on energy spectrum...}

2. S.G. Suprun, B.Ya. Moyzhes, Role of Electron correlations in formation of Pecar bipolaron // Fiz. Tverd. Tela. 24( 5), pp. 1571-1573 (1982).

3. S. I. Pekar. Research on Electron Theory in Crystals. Gostekhizdat, Moscow (1951), 256 p., (in Russian).

4. V.L. Vineckiy, M. S. Gitterman. To the theory of "added" charges interaction in polar crystals // Zh. Eksp. Teor. Fiz. 33(3), pp. 730-734 (1957).

5. V.L. Vineckiy, O. Meredov, V.A. Yanchuk, Quantum chemistry of bipolarons in the isotrop continuum environment // Teor. Eksp. Khim. 25(6), pp.641-647, (1989).

6. N.I. Kashirina, E.M. Mozdor, E.A. Pashickiy, V. I. Sheka, Bipolarons in anisotropic crystals //Izv. Ros. Akad. Nauk. Ser. Fiz. 59(8), pp. 127-133 (1995).

7. N.I. Kashirina, E.V. Mozdor, E.A. Pashitskij, V.I. Sheka, Bipolarons in anisotropic crystals and low dimensional structures // Semiconductor Physics Quantum electronics \& Optoelectronics 2(2), pp. 7-4 (1999).

8. V.K. Muhomorov, Ground state of an optical bipolaron (quasi- molecular dimer) with an intermediate strength of coupling // Opt. Spektrosk. 86(1), pp. 50-55 (1999).

9. V.K. Muhomorov, Bipolaron states of electron and magnetic properties of metal-ammonia systems // Phys. Stat. Sol. (b) 219(1), pp.71-89 (2000).

10. V.D. Lakhno, Salient features of ESR in the negatively charged clusters of water and ammonia IIIzv. Ros. Akad Nauk. Ser. Fiz. 61(9), pp.1827-1828 (1996)

11. E. P. Pokatilov, V. M. Fomin, J. T. Devreese, S. N. Balaban, S. N. Klimin, Bipolaron binding in quantum wiers // Phys. Rev.B 61(4), pp.2721-2728 (2000)
12. J.T. Devreese, V.M. Fomin, E.P. Pokatilov, E.A. Kotomin, R. Eglitis. Theory of bound polaron in the oxide compounds // Phys. Rev.B 63(18), pp.184304-1-184304-5 (2001).

13. J. Adamovski, M. Sobkowicz, B. Szafran, Electron pair in a Gaussian confining potential // Rev.B 62 (7) pp. 4234-4237 (2000).

14. S.J. Miyake, Strong coupling limit of the polaron ground state // J. Phys. Soc. Japan. 38(1), pp. 181-182 (1975).

15. J. Adamovski, S Bednarek. Stability of large bipolarons // J. Phys. Condens. Matter. 4(11), pp. 2845-2855 (1992).

16. H.L. Dewing, E.K.H. Salje, The effect of superconducting phase transition on the near-infrared absorption of $\mathrm{YBa}_{2} \mathrm{Cu}_{3} \mathrm{O}_{7-}$ delta // J. Phys. Supercond. Sci. Technol. 5(2), pp.50-53 (1992).

17. N.F. Mott, Polaron models of high-temperature superconductors //J.Phys.:Condens. Matter 5(22), pp. 3487-3506 (1993).

18. V. D. Lakhno, E. L. Nagaev, Magnetostriction ferrons //Fiz. Tverd. Tela, 20(1), pp.82-86 (1978).

19. V. D. Lakhno, Groung electron state in a field of coulomb impurity in the antiferromagnetic //Fiz. Tverd. Tela 27(3), pp.669-672 (1985).

20. G. Verbist, F. M. Peeters, J. T. Devreese, Large bipolarons in two and three dimantions // Phys. Rev. B 43(4), pp. 27122720 (1991).

21. V.L. Vineckiy, E.A. Pashickiy, Bipolaron states in crystals with anisotrop effective mass of free charge carriers // Fiz. Tverd. Tela 25(6), pp.1744-1747 (1983). 